\title{
Frecuencia y comportamiento epidemiológico e histológico en las neoplasias gastrointestinales asociadas a los pacientes portadores de VIH en el Hospital Juárez de México
}

\author{
Andy Gabriel Rivera Flores, ${ }^{1}$ Jaime Alberto González Angulo Rocha, ${ }^{1}$ Felipe Zamarripa-Dorsey, ${ }^{1}$ \\ Scherezada María Isabel Mejía Loza ${ }^{2}$
}

${ }^{1}$ Médico adscrito del servicio de Gastroenterología.

${ }^{2}$ Jefa del servicio de Gastroenterología.

Hospital Juárez de México. Ciudad de México, México.

Acta Gastroenterol Latinoam 2020;50(3):285-291

Recibido: 28/03/2020 / Aceptado: 15/08/2020 / Publicado online: 28/09/2020

\section{Resumen}

Antecedentes. El tracto gastrointestinal es uno de los sitios más comunes para el desarrollo de las neoplasias primarias en los pacientes con una infección preexistente por el virus de inmunodeficiencia humana (VIH). Aproximadamente el $40 \%$ de los pacientes con VIH desarrollará algún tipo de cáncer con una observación común: dichas neoplasias son más agresivas en estos pacientes. Solo el sarcoma de Kaposi y el linfoma no Hodgkin se localizan en el tracto gastrointestinal de manera primaria y se consideran como definitorios del
Correspondencia: Andy Gabriel Rivera Flores

Casino Casahonda, Belisario Dominguez Sec XVI, Tlalpan, CDMX,

México / Tel.: 005215517721399

Correo electrónico: andyrivera48@hotmail.com sindrome de inmunodeficiencia adquirida (SIDA). Existe otro grupo de neoplasias no definitorias del SIDA, pero que son más frecuentes en estos pacientes como la neoplasia anal y el cáncer colorrectal. Objetivos. Determinar la frecuencia, epidemiología e histología de las neoplasias gastrointestinales asociadas a los pacientes portadores de VIH. Material y métodos. Estudio descriptivo, de corte transversal y retrospectivo, realizado en el servicio de patología del Hospital Juárez de México del año 2006 al 2015. Los resultados fueron analizados con medidas de frecuencia relativas y centrales para la obtención de porcentajes, media, promedio y desviación estándar. Resultados. Se incluyeron a 75 pacientes con VIH asociado con algún cáncer gastrointestinal. La edad promedio fue de 35,4 $\pm 8,2$ años con predominio del género masculino en el $83 \%$. El sitio de localización más frecuente fue la zona del colon y el recto con el $43 \%$ de los casos. El tipo histológico predominante fue adenocarcinoma (35\%). Conclusiones. La neoplasia gastrointestinal en los pacientes portadores de VIH se evidencia con mayor frecuencia en el colon y el recto, siendo el tipo histológico más frecuente el adenocarcinoma.

Palabras claves. Neoplasias gastrointestinales, VIH, histología, SIDA. 


\section{Frequency, epidemiological and histological behavior in the gastrointestinal neoplasia associated with HIV carriers in the Hospital Juárez de México}

\section{Summary}

Background. The gastrointestinal tract is one of the most common sites for the development of primary neoplasms in the patients with a pre-existing infection with the human immunodeficiency virus (HIV). Approximately it is considered that the $40 \%$ of HIV patients will develop some type of cancer and there is a common observation that neoplasms are more aggressive in these patients. Only Kaposi's sarcoma and non-Hodgkin's lymphoma are located in the gastrointestinal tract in a primary way and are considered as defining acquired immunodeficiency syndrome (AIDS). There is another group of non-defining AIDS Neoplasm, but these are more frequent in these patients, such as anal and the colon neoplasia. Objectives. are to determine the frequency, epidemiology and histology of neoplasia's of gastrointestinal malignancies associated with patients with HIV. Material and methods. It was a descriptive, cross-sectional and retrospective study conducted in the pathology service of Hospital Juárez de México from 2006 to 2015. The results were analyzed with relative and central frequency measurements to obtain percentages, mean, average and deviation standard. Results. We included 75 HIV patients associated with a Gastrointestinal cancer. The average age was $35.4 \pm 8.2$ with predominance of the male gender in $83 \%$. The most frequent site of localization was the colon and the rectum with $43 \%$ of the cases. The service that most referred was oncology with 57\%. The predominant histological type was adenocarcinoma (35\%). Conclusions. Gastrointestinal neoplasia in patients with HIV is most frequently seen in the colon and the rectum, with adenocarcinoma being the most common histological type.

Key words. Gastrointestinal neoplasms, HIV, histology, AIDS.

\section{Abreviaturas}

VIH: Virus de inmunodeficiencia humana.

SK: Sarcoma de Kaposi.

LNH: Linfoma no Hodgkin.

CCR: Cáncer colorrectal.

SIDA: Sindrome de inmunodeficiencia adquirida.

TARV: Tratamiento antirretroviral.

NDS: Neoplasias definitorias de SIDA.

NNDS: Neoplasias no definitorias de SIDA.

HHV8: Herpes virus 8 humano.

VPH: Virus del papiloma humano.

IARC: Centro Internacional de Investigaciones sobre el Cáncer

\section{Introducción}

Desde los inicios de la epidemia del SIDA a principios de la década de los 80 , el cáncer ha figurado en un lugar destacado como una enfermedad concomitante. Las neoplasias malignas son una de las principales causas de hospitalización y muerte en la población con VIH positivo como consecuencia del uso del tratamiento antirretroviral y la disminución de las infecciones oportunistas. ${ }^{1,2}$ En la actualidad puede diagnosticarse un cáncer en el $25-40 \%$ de los pacientes con VIH. A diferencia de las épocas previas al TARV donde solo el 10\% de las muertes era de origen tumoral, ahora asciende hasta el $30 \%$, alcanzando tasas entre 1,1 y 1,8 cada 1000 pacientes por año de seguimiento. ${ }^{3,4}$

Las neoplasias gastrointestinales son muy frecuentes en la población general y podemos dividirlas en 2 grupos: las neoplasias definitorias de SIDA (NDS) en donde los dos máximos exponentes son el SK y el LNH, ambos incrementados paralelamente con la progresión de la inmunodeficiencia celular. ${ }^{5} 6$ En el segundo grupo están las neoplasias no definitorias del SIDA (NNDS), que en orden de frecuencia son el cáncer anal, el CCR, el gástrico y el hepatocarcinoma. Estas patologías han aumentado por la supervivencia que se consigue con el TARV $y$, además, se diagnostica en sujetos más jóvenes y con marcadores de mal pronóstico. ${ }^{7,8}$

En la etiopatogenia de las neoplasias gastrointestinales asociadas al VIH no existe un solo factor responsable de la aparición de las neoplasias definitorias o no del SIDA. El origen es multifactorial y, dentro de los posibles mecanismos implicados en su génesis, hay que considerar varios factores: el propio VIH, la inmunodepresión y el uso de TARV. 9,10

El VIH no es un virus oncogénico. Sin embargo, se han encontrado fragmentos de su genoma en algunos pacientes con el LNH y el gen tat parece estar implicado en el crecimiento de las células del SK. ${ }^{11,12}$

La inmunodepresión y la viremia del VIH no controlada aumentan el riesgo de desarrollar las neoplasias, lo que explicaría que el uso de TARV haya disminuido las NDS. ${ }^{13,14}$ Por otro lado, de forma paradójica, el incremento en las NNDS podría deberse a la mayor supervivencia de los pacientes con uso de TARV, pero la función inmune no se restaura completamente, condicionando una mayor exposición a los carcinógenos ambientales (el tabaco, la luz ultravioleta), una posibilidad más elevada de sobreinfección por los virus oncogénicos y un aumento de las mutaciones genéticas en los pacientes con historia familiar de cáncer. Además, la presencia del VIH origina un estímulo persistente de los linfocitos B, lo que altera la inmunidad antitumoral y facilita la capacidad oncogénica de los virus. ${ }^{15,16}$ 
El sarcoma de Kaposi (SK) es un tumor vascular asociado con la infección por el herpes virus 8 humano (HHV8) y la neoplasia más común entre los pacientes infectados por el VIH, afectando entre el 15 al 20\% de esta población. En una serie de casos, los órganos GI frecuentemente implicados fueron el intestino delgado (30\%), el colon (17\%) y el estómago (15\%). ${ }^{17}$

La neoplasia anal está en aumento y guarda relación con los estados crónicos de inmunodeficiencia y los genotipos oncogénicos del virus del papiloma humano (VPH) 16 y $18 .{ }^{18-20} \mathrm{Su}$ prevalencia en los varones homosexuales es 35 veces superior a la observada en la población general. En el $90 \%$ de los casos es un carcinoma escamoso y los síntomas son dolor local y tenesmo. En ocasiones pueden detectarse masas o nódulos en la zona anal. Es importante destacar que rara vez se realiza en la consulta un tacto rectal de los pacientes con VIH durante sus revisiones periódicas. Por ello, habría que estudiar la rentabilidad costo-beneficio de la incorporación, no solo del tacto rectal, sino también de la inspección anal y perianal, de la citología anal y del cribado del VPH para detectar su presencia en todos los pacientes VIH positivo con o sin síntomas. ${ }^{21-23}$

En el caso del CCR, en la mayoría de las ocasiones, el diagnóstico se hace con una situación clínica desfavorable y con metástasis. El adenocarcinoma de colon, que representa el $85 \%$ de las neoplasias de esta localización, suele presentarse como una enfermedad diseminada, con una supervivencia media inferior a los 6 meses en los pacientes con VIH. Se considera que es una coincidencia diagnóstica más que una relación directa, aunque hay casos en los que se entiende que la inmunodepresión juega un papel importante en su génesis. ${ }^{24,25}$

El hepatocarcinoma en los enfermos portadores de VIH se asocia a la infección por el virus de la hepatitis $\mathrm{B}$ o C, la existencia de cirrosis, el consumo de alcohol y tabaco. ${ }^{26}$ Antes del TARV no hubo un incremento del hepatocarcinoma en los pacientes con VIH, muy probablemente porque perecían a temprana edad. Hoy en día, la hepatopatía ocupa un lugar preponderante dentro de las causas de muerte en todas las series de enfermos con VIH. ${ }^{27}$ Por este motivo, no es de extrañar que en pocos años se prevea un incremento en el diagnóstico del carcinoma hepatocelular en aquellos sujetos en los que el TARV haya conseguido aumentar su supervivencia. ${ }^{28,29}$

El tratamiento de las neoplasias gastrointestinales se basa en tres pilares fundamentales: el tratamiento de la propia neoplasia, que variará según la naturaleza de la misma; el uso de TARV, la profilaxis y el tratamiento de las enfermedades oportunistas. ${ }^{30}$ El procedimiento es proporcionar al enfermo el mismo tipo de tratamiento que en los individuos VIH negativos, siempre que su estado general lo permita. El pronóstico en la mayoría de las ocasiones va a venir determinado por factores relacionados con la propia neoplasia más que por la infección del VIH. ${ }^{31,32}$

En este contexto, el objetivo principal de este trabajo fue determinar la frecuencia, la epidemiología e la histología de las neoplasias gastrointestinales asociadas a los pacientes portadores del VIH.

\section{Material y métodos}

Se efectuó un estudio retrospectivo y descriptivo del año 2006 al 2015, realizado en el servicio de Gastroenterología del Hospital Juárez de México en cooperación con el servicio de anatomía patológica e infectología. Dicho centro es un hospital universitario de tercer nivel. Los datos de los pacientes fueron extraídos del reporte de anatomía patológica. Posteriormente, se realizó una búsqueda en los expedientes o en las computadoras del servicio de infectología para obtener los valores de CD4 seleccionando solamente los pacientes que cumplieran con los siguientes criterios de inclusión: pacientes con VIH con reporte histopatológico de malignidad; que tuvieran conteo de CD4 en su expediente o en la computadora del servicio de infectología y que hubieran notas acerca del tratamiento antirretroviral; mayores de 18 años. Se excluyeron: los pacientes con VIH con reporte histopatológico de patologías benignas (pólipos, enfermedad inflamatoria intestinal, infecciones oportunistas, etc.); que no tuvieran conteo de CD4 ni registro del tratamiento antirretroviral (Figura 1). Los datos de los pacientes que cumplieron con los criterios fueron analizados en Excel y SPSS V 23 para la obtención de medidas de frecuencia relativas y centrales, porcentajes, media, promedio y desviación estándar.

La realización de este trabajo fue aceptada y autorizada por el Comité de ética y de ensayos clínicos del Hospital Juárez de México y no tuvo ningún patrocinio, financiamiento ni conflicto de intereses.

\section{Resultados}

Se incluyeron a 75 pacientes con VIH asociado con algún tipo de cáncer gastrointestinal. La edad promedio fue de 35,4 $\pm 8,2$ años con predominio del género masculino en el 83\% (Tabla 1). El sitio de localización más frecuente fue el colon y el recto con el $43 \%$ de los casos, seguido del estómago con el $20 \%$; el ano y el esófago con $16 \%$ cada uno (Tabla 2). El servicio que más refirió fue oncología con el $57 \%$. El tipo histológico predominante fue adenocarcinoma $(35 \%)$, seguido del LNH (26\%), el sarcoma de Kaposi (24\%) y el 


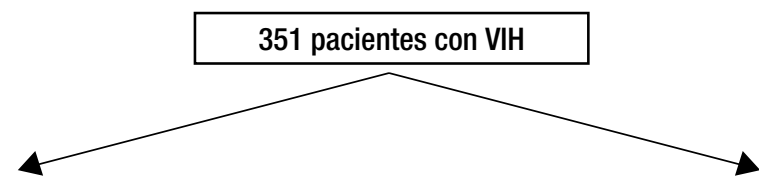

Criterios de inclusión: pacientes con diagnóstico de VIH y reporte histopatológico de malignidad en el tracto gastrointestinal; que tuvieran CD4 en su expediente y que, además, tuvieran registro del tratamiento antirretroviral.

$$
\mathrm{n}=75
$$

Pacientes incluidos en el estudio:

75 pacientes tuvieron reporte histopatológico de malignidad en el tracto gastrointestinal.

\section{Neoplasias del tracto gastrointestinal}

$$
(n=75) \text { : }
$$

Esófago (12)

Estómago (15)

Intestino delgado (4)

Colon y recto (32)

$$
\text { Ano (12) }
$$

Criterios de exclusión: pacientes con VIH con reportes de patología negativos para malignidad (infecciones oportunistas, pólipos, infecciones bacterianas, víricas o parasitarias, enfermedad inflamatoria intestinal, colitis indeterminada); que no tuvieran CD4 o nota de tratamiento antirretroviral.

$$
\mathrm{n}=\mathbf{2 7 6}
$$

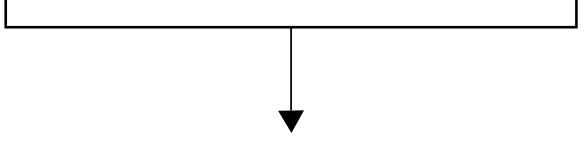

Pacientes excluidos o eliminados:

276 con estos hallazgos histopatológicos:

- 67 infecciones oportunistas;

- 18 enfermedad inflamatoria intestinal;

- 28 infecciones bacterianas;

- 28 infecciones parasitarias;

- 47 colitis indeterminadas;

- 42 infecciones víricas;

- 36 no tenían CD4 ni registro de tratamiento antirretroviral;

- 10 pólipos benignos.

Tabla 1. Caracteristicas demográficas de los pacientes con el VIH y neoplasias del tracto gastrointestinal

\begin{tabular}{lc}
\hline Variable & $\mathrm{n}=75$ \\
\hline Género & $62(83 \%)$ \\
Hombres & $13(17 \%)$ \\
Mujeres & \\
Edad & $35,4( \pm 8,2)$ \\
Servicio de referencia & \\
1. Oncología & $43(57 \%)$ \\
2. Gastroenterología & $1(1 \%)$ \\
3. Endoscopía & $31(42 \%)$ \\
& \\
Conteo de CD4 & \\
$<400$ mm $^{3}$ & $38(51 \%)$ \\
$>400$ mm $^{3}$ & $37(47 \%)$ \\
Tratamiento antiretroviral & \\
Sí & \\
No & $37(47 \%)$ \\
Abandono & $10(13 \%)$ \\
Diagnóstico nuevo & $10(13 \%)$ \\
\hline
\end{tabular}


epidermoide (15\%). Todos los LNH de células B y los sarcoma de Kaposi tenían CD4 menor a $400 \mathrm{~mm}^{3}$ y ninguno tenía un tratamiento adecuado antirretroviral (Tabla 3 y 4 ).

\section{Discusión y conclusiones}

A nivel mundial en la población general sin VIH, según datos del Centro Internacional de Investigaciones sobre el Cáncer (IARC), las neoplasias gastrointestinales

Tabla 2. Asociación entre las características demográficas y el tipo histológico de las neoplasias gastrointestinales asociadas al VIH

\begin{tabular}{|c|c|c|c|c|c|}
\hline Variables & $\begin{array}{c}\text { Adenocarcinoma } \\
\text { (26) }\end{array}$ & $\begin{array}{l}\text { LNH de células B } \\
\qquad(20)\end{array}$ & $\begin{array}{l}\text { SK } \\
(18)\end{array}$ & $\begin{array}{c}\text { Epidermoide } \\
\text { (11) }\end{array}$ & $\begin{array}{l}\text { Total } \\
(75)\end{array}$ \\
\hline Hombres & $19(73 \%)$ & $17(85 \%)$ & $18(100 \%)$ & $8(73 \%)$ & $62(83 \%)$ \\
\hline Mujeres & $7(27 \%)$ & $3(15 \%)$ & 0 & $3(27 \%)$ & $13(17 \%)$ \\
\hline $\mathrm{CD} 4<400 \mathrm{~mm}^{3}$ & 0 & $20(100 \%)$ & 18 & 0 & 38 (51\%) \\
\hline $\mathrm{CD} 4>400 \mathrm{~mm}^{3}$ & $26(100 \%)$ & 0 & 0 & 11 & $35(49 \%)$ \\
\hline \multicolumn{6}{|c|}{ Tratamiento antirretroviral } \\
\hline Sí & $26(100 \%)$ & 0 & 0 & 11 & 37 (49\%) \\
\hline No & 0 & $4(20 \%)$ & $6(33 \%)$ & 0 & $10(13,5)$ \\
\hline Abandono & 0 & $10(50 \%)$ & 0 & 0 & $10(13,5 \%)$ \\
\hline Diagnóstico nuevo & 0 & $6(30 \%)$ & 12 (67\%) & 0 & $18(24 \%)$ \\
\hline Total pacientes & 26 & 20 & 18 & 11 & 75 \\
\hline
\end{tabular}

LNH: Linfoma no Hodgkin; SK: Sarcoma de Kaposi.

Tabla 3. Localización e histologia de las neoplasias gastrointestinales en pacientes con el VIH

\begin{tabular}{cc}
\hline Localización & Histología \\
\hline Esófago (12) & Adenocarcinoma (6) \\
& Epidermoide (3) \\
& SK (3) \\
Estómago (15) & LNH de células b (6) \\
& Adenocarcinoma (6) \\
SK (3) \\
Intestino delgado (4) \\
SK en la primera porción del duodeno (2) \\
SK en la segunda porción del duodeno (2) \\
Ano (12) \\
Adenocarcinoma (14) \\
LNH células b (14) \\
SK (4) \\
\\
Total & Epidermoide (8) \\
& SK (4) \\
& 75
\end{tabular}

Tabla 4. Histología de las neoplasias gastrointestinales en pacientes con el VIH

\begin{tabular}{cc}
\hline Histología & Localización \\
\hline Adenocarcinoma (26) & Colón (14) \\
& Estómago (6) \\
Esófago (6) & Colon (14) \\
LNH de células B (20) & Estómago (6) \\
& Ano (4) \\
SK (18) & Colon (4) \\
& Intestino delgado (4) \\
& Estómago (3) \\
& Esófago (3) \\
& \\
Epidermoide (11) & Ano (8) \\
& Esófago (4) \\
Total & 75
\end{tabular}

LNH: Linfoma No Hodgkin; SK: Sarcoma de Kaposi. 
con mayor mortalidad entre el año 2018 y 2019 fueron el CCR, la gástrica y la hepática. En México el CCR es el segundo en frecuencia en la población masculina y el quinto en la población femenina; por lo cual, es de vital importancia reconocer que existe una limitada información respecto a la epidemiología de las neoplasias gastrointestinales en los pacientes con VIH. ${ }^{33}$ Estudios demuestran que las neoplasias más frecuentes en estos pacientes son dentro de las definitorias: el SK y el LNH, lo cual coincidió con los resultados de nuestro estudio. Sin embargo, fueron más frecuente el LNH (26\%) y el SK (24\%). En las neoplasias no definitorias, las más frecuentes mencionadas en la literatura son las del canal anal y el colón. En nuestro estudio llama la atención que fue más prevalentes el cáncer de colón (43\%); seguido por el de estómago con un $20 \%$ y el canal anal fue igual que el del esófago (16\%). Esto puede ir de la mano a que en México el CCR (según estudios del IARC) es el segundo más frecuente en hombres y nuestra población era masculina en su mayoría. No obstante, desafortunadamente no se hizo énfasis en los antecedentes heredofamiliares de estos pacientes (síndrome de Lynch, síndromes polipósicos, etc.) ya que muchos de los datos de estos pacientes se extrajeron del CD4 en la computadora del servicio de infectología. ${ }^{3,7,33}$

En cuanto a la histología, el tipo histológico más frecuente fue el adenocarcinoma (35\%), seguido del LNH (26\%), del sarcoma de Kaposi (24\%) y del epidermoide $(15 \%)$. Aunque hay muy pocos estudios acerca de la histopatología, la mayoría coincide que el tipo más frecuente es el adenocarcinoma, como afirma nuestro trabajo. ${ }^{24,25}$

En México llama la atención que el 49\% tomaba TARV y que de estos pacientes todos tuvieron el tipo histológico adenocarcinoma y epidermoide, mientras que los pacientes sin tratamiento o con abandono todos tuvieron el SK y el LNH. Estos datos coinciden con la literatura mundial en que los pacientes con TARV tienen mayor esperanza de vida y, por ende, desarrollan el adenocarcinoma o el epidermoide contrario a los pacientes con la enfermedad definitoria del SIDA. ${ }^{31,32}$

\section{Conclusiones}

En nuestro estudio evidenciamos que la neoplasia gastrointestinal con mayor frecuencia fue el CCR, siendo el tipo histológico más frecuente el adenocarcinoma. El reconocer estas patologías en el paciente inmunodeprimido es de suma importancia para el abordaje diagnóstico y terapéutico temprano. Sugerimos se realicen trabajos multicéntricos para poder tener una mayor cantidad de información estadística acerca de esta patología, ya que es muy limitada a nivel mundial.

\section{Sostén económico o patrocinio. Ninguno. \\ Conflicto de intereses. Ninguno.}

\section{Referencias}

1. Gottlieb MS, Schroff R, Schanker HM, Weisman, JD, Fan PT, Wolf RA, Saxon A. Pneumocystis carinii pneumonia and mucosal candidiasis in previously healthy homosexual men: evidence of a new acquired cellular immunodeficiency. N Engl J Med 1981; 305 (24): 1425-1431.

2. Herida M, Mary-Krauze M, Kaphna R, et al. Incidence of nonAIDS-defining cancers before and during the highly active antiretroviral therapy era in a cohort of human immunodeficiency virus-infected patients. J Clin Oncol 2003; 21 (18): 3447-3453.

3. Long JL, Engels EA, Moore RD, Gebo KA. Incidence and outcomes of malignancy in the HAART era in an urban cohort of HIVinfected individuals. AIDS 2008; 22 (4): 489-496.

4. Bonnet F, Lewden Ch, May Th, et al. Malignancy-related causes of death in human immunodeficiency virus-infected patients in the era of highly active antiretroviral therapy. Cancer 2004; 101 (2): 317-324.

5. Simard EP, Engels EA. Cancer as cause of death among people with AIDS in the United States. Clin Infect Dis 2010; 51 (8): 957-962.

6. Hensel M, Goetzenich A, Lutz T, Sroehr A, Moll A, Rockstroh J, Hanhoff N, Jäger H, Mosthaf F. HIV and cancer in Germany. Dtsch Arzlebl Int 2011; 108 (8): 117-122.

7. Stebbing J, Krown SE, Bower M, Batra A, Slater S, Serraino D, Dezube BJ, Dhir AA, Pantanowitz L. Primary esophageal carcinoma in the era of highly active antiretroviral therapy. Arch Intern Med 2010; 170 (2): 203-207.

8. Alfa-Wali M, Tait D, Allen-Mersch T, Tekkis P, Nelson M, Stebbing J, Antoniou A, Bower M. Colorectal cancer in HIV positive individuals: The immunological effects of treatment. Eur J Cancer 2011; 47 (16): 2403-2407.

9. Pantanowitz L. Overview of non-AIDS-defining malignancies in HIV infection. Section Editor: Dezeube BJ; Deputy Editor: Ross ME. 2020. Disponible en: www.uptodate.com

10. Lim ST, Levine AM. Non-AIDS-defining cancers and HIV infection. Curr HIV/AIDS Rep 2005; 2 (3): 146-153.

11. Ensoli B, Barillari G, Salahuddin SZ, Gallo RC, Wong-Staal F. Tat protein of HIV-1 stimulates growth of cells derived from Kaposi's sarcoma lesions of AIDS patients. Nature 1990; 345 (6270): 84-86.

12. Albini A, Barillari G, Benelli R, Gallo RC, Ensoli B. Angiogenic properties of human immunodeficiency virus type 1 Tat protein. Proc Natl Acad Sci USA 1995; 92 (11): 4838-4842.

13. Kesselring A, Gras L, Smit C, van Twillert G, Verbon A, de Wolf F, Reiss P, Wit F. Immunodeficiency as a risk factor for nonAIDS-defining malignancies in HIV-1-infected patients receiving combination antiretroviral therapy. Clin Infect Dis 2011; 52 (12): 1458-1465.

14. Reekie J, Gatell JM, Yust I, et al. Fatal and non-fatal AIDS and nonAIDS events in HIV-1 positive individuals with high CD4 counts according to viral load strata. AIDS 2011; 25 (18): 2259-2268. 
15. Silverberg M, Leyden W, Chao C, Xu L, et al. Infection-related Non-AIDS-defining risk in HIV-infected and uninfected persons: Increased Rates in HIV+ vs HIV-negatives in Kaiser. Conference on Retroviruses and Opporunistic Infections. Montreal, Canada 2009.

16. Riva G, Barozzi P, Torelli G, Luppi M. Immunological and inflammatory features of Kaposi's sarcoma and other Kapose's sarcoma-associated herpesvirus/human herpesvirus 8-associated neoplasias. AIDS Rev 2010; 12 (1): 40-51.

17. Ioachim HL, Adsay V, Giancotti FR, Dorsett B, Melamed J. Kaposi's sarcoma of internal organs. A multiparameter study of 86 cases. Cancer 1995; 75 (6): 1376-1385.

18. Palefsky J. Human papillomavirus-related disease in people with HIV. Curr Opin HIV AIDS 2009; 4: 52-56.

19. Chaturvedi AK, Madeleine MM, Biggar RJ, Engels EA. Risk of human papillomavirus-associated cancers among persons with AIDS. J Natl Cancer Inst 2009; 101 (16): 1120-1130.

20. Parés D, Mullerat J, Pera M. Neoplasia intraepitelial anal. Med Clin (Barc) 2006; 127 (19): 749-755.

21. Palefsky JM. Anal cancer prevention in HIV-positive men and women. Curr Opin Oncol 2009; 21 (5): 433-438.

22. Palefsky JM, Giuliano AR, Goldstone $S$, et al. HPV vaccine against HPV infection and anal intraepithelial neoplasia. N Engl J Med 2011; 365 (17): 1576-1585.

23. Wilkin T, Lee JY, Lensing SY, et al. Safety and immunogenicity of the quadrivalent human papillomavirus vaccine in HIV-1- infected men. J Infect Dis 2010; 202 (8): 1246-1253.

24. Shiels MS, Cole SR, Kirk GD, Poole C. A meta-analysis of the incidence of non-AIDS cancers in HIV-infected individuals. J Acquir Immune Defic Syndr 2009; 52 (5): 611-622.

25. Patel P, Hanson DL, Sullivan PS, Novak RM, Moorman AC, Tong TC, Holmberg SD, Brooks J; Adult and Adolescent Spectrum of Disease Project and HIV Outpatient Study Investigators. Incidence of types of cancer among HIV-infected persons compared with the general population in the United States, 1992-2003. Ann Intern Med 2008; 148 (10): 728-736.
26. Guzmán-Fulgencio M, Berenguer J, Fernandez de Castro I, et al. Sustained virological response to interferon- $\alpha$ plus ribavirin decreases inflammation and endothelial dysfunction markers in HIV/HCV co-infected patients. J Antimicrob Chemother 2011; 66 (3): 645-649.

27. Di Benedetto F, De Ruvo N, Berretta M, Masetti M, Montaldi R, Di Sandro S, Ballarin R, Codeluppi M, Guaraldi G, Gerunda GE. Hepatocellular carcinoma in HIV patients treated by liver transplantation. Eur J Surg Oncol 2008; 34 (4): 422-427.

28. Rafael-Valdivia L, Miró JM, Rimola A. Trasplante hepático en pacientes con infección por VIH. Gastroenterol Hepatol 2010; 33 (9): 660-669.

29. Salhab M, Canelo R. An overview of evidence-based management of hepatocellular carcinoma: a meta-analysis. J Cancer Res Ther 2011; 7 (4): 463-475.

30. Engels EA. Non-AIDS defining malignancies in HIV-infected persons: etiologic puzzles, epidemiologic perils, prevention opportunities. AIDS 2009; 23 (8): 875-885.

31. Powles T, Robinson D, Stebbing J, Shamash J, Nelson M, Gazzard B, Mandelia S, Møller H, Bower M. Highly active antiretroviral therapy and the incidence of non-AIDS-defining cancers in people with HIV infection. J Clin Oncol 2009; 27 (6): 884-890.

32. Silverberg MJ, Abrams DI. Do antiretrovirals reduce the risk of nonAIDS defining malignancies? Curr Opin HIV AIDS 2009; 4: 42-51.

33. Latest global cancer data: Cancer burden rises to 18.1 million new cases and 9.6 million cancer deaths in 2018. International Agency for Research on Cancer 2018. Comunicado de prensa No 263. Consultado en http://www.iarc.fr/en/media-centre/pr/2018/pdfs/ pr263_E.pdf 